\title{
Theory of Allocating and Scheduling Resources at Airport Passenger
}

\section{Terminals: A Review}

\section{Shaowu Cheng ${ }^{1, a}$, Yaping Zhang ${ }^{2, b}$ and Yuanyuan Guo ${ }^{2, c}$}

\author{
${ }^{1}$ Department of Traffic Information and Control Engineering, Harbin Institute of Technology, Harbin, \\ China, 150006 \\ ${ }^{2}$ Department of Traffic Engineering, Harbin Institute of Technology, Harbin, China, 150006, \\ acsw_h@hit.edu.cn, bzxlt0905@163.com, ‘hitgyy@126.com
}

Keywords: Allocating and Scheduling, Resource, Airport Passenger Terminal

\begin{abstract}
This paper reviews theory of allocating and scheduling resources at airport passenger terminals, from three aspects, that are method of integrating airport operational data, method of predicting passenger flow at airport terminals and optimization method of allocating and scheduling resources at airport terminals. Directions and challenges of future research are identified.
\end{abstract}

\section{Introduction}

The guarantee of flight safety and punctuality is the basic requirements of the civil aviation convenient transportation, the United States established a clear development goals aiming at the efficiency of air transport in the Next Generation Air Transport System (NextGen): travelers can take off after reaching the terminal within 30 minutes except in very bad weather conditions. In 2010, China's civil aviation passenger traffic exceeded 260 million, ranking in the world second. Management of airport terminals is becoming more and more important.

On one hand, in large hub airport terminals, for passenger traffic increased rapidly, long stranded passengers in check-in, security and other areas can easily lead to group events, which bring a great deal of negative impact to the civil aviation industry. Reduce the residence time in the airport terminal building has two ways: first, increase the terminal passenger service resources; second, according to passenger flow fluctuation at different times, take a reasonable allocation and scheduling of the terminal passenger service resources. Under the premise of not increasing existing resources, the latter is an effective way to resolve the problem of passengers long time stuck.

On the other hand, in the small airport terminal, due to a lack of passenger traffic, coupled with resource allocation and scheduling without considering passenger flow fluctuation, passenger service resources is idle in some periods, which artificially reduced the use efficiency of passenger service resources and increase operational costs of airport terminals. According to passenger flow fluctuation at different periods, taking a reasonable allocation and scheduling of the terminal passenger service resources benefits reducing the operational costs of the airport terminal, and improving the operational efficiency of the airport.

The United States has started to study theoretical problem of the intelligent allocation and scheduling of terminal passenger service resources [1,2]. China just started the research in this field [3]. At present, theoretical research in intelligent allocation and scheduling of the terminal resources fall into three fields: first, method of integrating airport operation data, second, method of predicting passenger flow at airport passenger terminals, third, optimization method of allocating 
and scheduling passenger service resource at airport terminals. This paper reviews the theoretical study on these three fields, and identifies directions and challenges of future research in the field of intelligent allocation and scheduling of terminal resource.

\section{Method of integrating airport operation data}

In aspect of integrating airport operation data, research focus on the airport information systems integration technology for ensuring safety and efficiency of airport operations. The U.S. Department of Transportation provides guidance for the development of integrated airport information system through the airport cooperative research program [4]. Shen Yang proposed the multi-layer distributed database architecture for integrating airport operation data, based on Web Service and the message queue [5]. Li Ming etc. studied the technology of airport data exchange platform in a distribution heterogeneous environment [6].

The above studies involved in framework of airport information systems, architecture of airport operations database and technology of airport information systems integration, but didn't involve method of needs analysis and logical modeling aiming at data integration for smart allocation and scheduling the airport passenger service resources. Therefore, it is difficult to get the accurate data demand from intelligent allocation and scheduling airport passenger service resources, also difficult to guarantee the logic rationality and query efficiency of data integration. The data integration methods, oriented terminal passenger services resources intelligent allocation and scheduling, in data integration needs analysis and logical modeling method centered, needs to be studied.

\section{Method of predicting airport terminal passenger flow}

Evaluation index of airport terminal operation efficiency and service level include available space for per passenger, passenger queuing time in a key part of business process and the total time required by a passenger departure process. Values of these indices are determined by the variation of airport terminal passenger flow. Accurately predicting airport terminal passenger flow and reasonably allocating and scheduling terminal passenger service resources in accordance with the terminal passenger flow fluctuations, can ensure that values of these indices are not affected by passenger flow fluctuations. Therefore, airport terminal passenger flow is basis of allocating and scheduling terminal passenger service resources.

At present, there are two methods forecasting the airport terminal passenger flow. One method is based on the data obtained from manual counts [7], using of mathematical statistical methods to obtain the probability density function of passenger's stranded time at airport terminal, and to estimate passenger number reaching at terminal in different periods of a day. Another method use flight scheduling data, check-in passenger's number distribution, and flight operations data, by simple data statistics, to establish passenger flow distribution model with one week cycle [8]. The two forecasting methods are based on the assumption that passenger flow distribution repeats at constant cycle.

In fact, with air transport demand change, passenger flow changes on the time scale of the year, seasons, months, weeks, days and hours, has not only periodicity but also randomness. The passenger flow forecasting method based on assumption that passenger flow distribution cyclically changes, ignores randomness of terminal passenger flow changes, and cannot reflect the complexity of terminal passenger flow fluctuation. Research is required for prediction method of airport terminal passenger flow, considering both periodicity and randomness characteristics of airport terminal passenger flow fluctuation. 


\section{Optimization method of allocating and scheduling resource of airport passenger terminals}

Research on terminal passenger service resource allocation and scheduling focus on resource scheduling problem of a part of airport business process (such as parking bays scheduling [9], check-in counter scheduling [10], baggage carousels scheduling [11]) and the optimization methods of key index about airport terminal operations service levels (such as lower check-in aspects waiting time of passengers [12], reducing the boarding process time [13]).

In fact, simply optimizing a part of passenger service process, often results in blockage of the downstream parts [14], for example, too fast passenger flow at check-in areas will result in traveler strand at security control areas. Therefore, the optimization of allocation and scheduling resources should consider airport business process as a whole. At present, optimization methods of terminal passenger service resource allocation and scheduling include mathematical programming method and process simulation method. Application scope of mathematical programming method is restricted, because it assumes that passenger flow satisfies some probability distribution, and requires necessary simplification of the model, [10]. Therefore, process simulation and optimization, with target to improve overall performance of terminal passenger service process, become a main optimization method of allocating and scheduling airport terminal resources $[15,16]$.

At present, the process simulation and optimization method to improve overall performance of airport terminal passenger service process requires a number of simulation test, with parameter values of each simulation test set by person's experience, and with lack of scientific basis. In addition, it is impossible for process simulation to traverse all possible combination of resources, because of the "combinatorial explosion" problem. Therefore, it is necessary to develop an intelligent method of setting simulation parameter values, and to establish an intelligent optimization method of allocating and scheduling airport terminal resources.

\section{Directions of future research}

The main research direction of airport terminal resource allocation and scheduling theory is to factor improvement of passenger service levels and airport terminal operation efficiency, airport data integration, passenger flow forecasting and baggage demand prediction, resource allocation and scheduling, as well as passenger service process simulation and optimization into a unified theoretical framework, and to reveal quantitative relationship among airport operational information, passenger flow, baggage demand, resource allocation and scheduling, passenger service levels and operational efficiency, and establish a theory system of intelligent allocation and scheduling of airport terminal resources, with airport operational data integration on a basis, and with passenger flow forecasting and passenger service process simulation and optimization as a core technology. To achieve the above objectives, research for following theoretical issues is required.

Data integration method of intelligent allocation and scheduling of airport passenger terminal resources. According to data demand of passenger flow prediction and business process simulation \& optimization, taking into account efficiency of data querying and future expansion capabilities of data integration, build the logic model of data integration for intelligent allocation and scheduling of airport passenger terminal resources, using data warehouse modeling theory, verify the logical model and improve it by testing data query, and lay theoretical foundation for implementing a data warehouse used to allocate and schedule airport passenger terminal resources. 
Information fusion method of predicting passenger flow at airport terminals. Predict variation of passenger flow at airport passenger terminals, fusing ticket booking information and passenger flow monitoring information, based on nonlinear modeling theory such as BP neural network, theory of chaotic time series etc.

Warning method of passenger flow anomaly at airport terminals. Set up a 'normal' value for passenger flow at distinctive period, and then difference between the real-time monitoring passenger flow and the "normal" value of passenger flow at corresponding period, is used as indicators to evaluate state of passenger flow, and determine threshold of passenger flow anomaly by experiment method.

Prediction method of passenger baggage demand. Airport terminal passenger baggage demand is closely related to passenger flow at airport terminals. Using passenger flow monitoring data and passenger baggage historical data obtained from departure system as data of training neural network, establish a BP neural network model to predict passenger baggage demand.

Intelligent simulation optimization method of airport passenger terminal resource allocation and scheduling. Based on dyeing time Petri net, describe logical relationship and information flow among business activities, allocation and scheduling plan of resources used by business activities at airport terminals, as well as coordination mechanism between passenger departure process and baggage handling process. Design discrete event simulation algorithm of passenger service process at airport terminals, using the Three-Phase approach. Optimize resource allocation and scheduling plan for each simulation run, based on genetic algorithm.

\section{Remarks and conclusion}

This paper reviews theory of allocating and scheduling resources at airport passenger terminals, from three aspects, that are method of integrating airport operational data, method of predicting passenger flow at airport terminals and optimization method of allocating and scheduling resources at airport terminals. The main direction of future research is to factor improvement of passenger service level and airport terminal operation efficiency, airport operational data integration, passenger flow prediction and baggage demand prediction, resource allocation and scheduling, as well as passenger service process simulation and optimization into a unified theoretical framework, and establish a theory system of intelligent allocation and scheduling of airport terminal resources, with airport operational data integration on a basis, and with passenger flow forecasting and passenger service process simulation and optimization as a core technology. Challenges of future research include:

(1) How to describe data demand of airport terminal passenger flow prediction and business process simulation \& optimization, and build logical data model to meet the demand.

(2) How to build quantitative relationship among passenger flow fluctuation, flight ticket booking information, flight scheduling, and historical passenger flow information.

(3) How to identify disturbance factors and cycle of passenger flow fluctuation, and determine threshold of potential passenger flow anomaly.

(4) How to describe collaborative mechanisms among passenger service processes and build a formal model of resource allocation and scheduling plan, and how to design intelligent simulation optimization algorithm for airport passenger terminal resource allocation and scheduling. 


\section{Acknowledgements}

This research is funded by National Natural Science Foundation of China (Grant No. 61179069) and Heilongjiang Province Natural Science Foundation of China (Grant No. E201114).

\section{References}

[1] F. Jaehn, Solving the flight gate assignment problem using dynamic programming, Zeitschrift für Betriebswirtschaft, 10(2010) 1027-1039.

[2] M. Saffarzadeh, J.P. Braaksma, Optimum design and operation of airport passenger terminal buildings, Journal of the Transportation Research Board, 1703(2000) 72-82.

[3] M. Ehrgott, B. Naujoks, T.J. Stewart, J. Wallenius(eds.), Multiple Criteria Decision Making for Sustainable Energy and Transportation Systems, $1^{\text {st }}$ ed., Springer, Berlin, 2010.

[4] C. Stocking, J. DeLong, V. Braunagel. T. Healy and S. Loper, Analysis and Recommendations for Developing Integrated Airport Information Systems, Contractor's Final Report for ACRP Project 1-03, Submitted (2008).

[5] Y. Shen, Web service-based architecture of airport operation database system, Journal of Value Engineering (in Chinese), 5(2011) 154-155.

[6] M. Li, S.Y. Zhang, X.J. Zhao, Design and implementation of information integration for airport based on data exchanging platform, Journal of Science Technology and Engineering(in Chinese), 20(2009) 6235-6238.

[7] K. Wonkyu, P. Yonghwa, J.K. Byung, Estimating hourly variations in passenger volume at airports using dwelling time distributions, Journal of Air Transport Management, 10(2004) 395-400.

[8] C.V. Robertson, S. Shrader, D.R. Pendergraft, L.M. Johnson, K.S. Silbert, The role of modeling demand in process re-engineering, in: Proceedings of the 2002 Winter Simulation Conference(WSC'02), IEEE Computer Society Press, New York, 2002, pp.1454-1458

[9] S.Y. Yan, C.H. Tang, A heuristic approach for airport gate assignments for stochastic flight delays, European Journal of Operational Research, 2(2007) 547-567.

[10]C.Y. Liu, Z.N. Deng, J.J. Zhang, Research on check-in problem in airport terminal building, in: Proceedings of 2007 Control and Decision Annual Conference of China(in Chinese), Control and Decision Press, Shenyang, China, 2007, pp.789-792.

[11]X. Lu, J.F. Zhu, X.W. Tang, Airport Luggage process modeling and simulation, Journal of System Simulation, 14(2008) 3876-3880.

[12]C.A. Chung, T. Sodeinde, Simultaneous service approach for reducing air passenger queue time. Journal of Transportation Engineering, 1(2000) 85-88.

[13]H.Van Landeghem, A. Beuselinck, Reducing passenger boarding time in airplanes: A simulation based approach, European Journal of Operational Research, 2(2002) 294-308.

[14]I.E. Manataki, K.G. Zografos, Assessing airport terminal performance using a system dynamics model, Journal of Air Transport Management, 16 (2010) 86-93.

[15]Liene Freivalde, Lelde Lace, Improvement of passenger flow management in an airport terminal, in: Proceedings of the 5th international Science Conference Business and Management, Vilnius, Lithuania, 2008, pp.659-664.

[16]K. C. James, Performance improvement studies of an airport terminal using discrete-event simulation, Journal of Computer Modeling and New Technologies, 3(2009) 58-64. 